\title{
Testing the itinerancy of spin dynamics in superconducting $\mathrm{Bi}_{2} \mathrm{Sr}_{2} \mathrm{CaCu}_{2} \mathrm{O}_{8+\delta}$
}

\author{
Guangyong Xu' ${ }^{1}$, G. D. Gu' ${ }^{1}$ M. Hücker ${ }^{1}$, B. Fauqué2 ${ }^{2}$ T. G. Perring ${ }^{3,4}$, L. P. Regnault ${ }^{5}$ \\ and J. M. Tranquada ${ }^{1 \star}$
}

\begin{abstract}
Much of what we know about the electronic states of hightemperature superconductors is due to photoemission ${ }^{1-3}$ and scanning tunnelling spectroscopy ${ }^{4,5}$ studies of the compound $\mathrm{Bi}_{2} \mathrm{Sr}_{2} \mathrm{CaCu}_{2} \mathrm{O}_{8+\delta}$. The demonstration of well-defined quasiparticles in the superconducting state has encouraged many theorists to apply the conventional theory of metals, Fermiliquid theory, to the cuprates ${ }^{6-9}$. In particular, the spin excitations observed by neutron scattering at energies below twice the superconducting gap energy are commonly believed to correspond to an excitonic state involving itinerant electrons ${ }^{10-14}$. Here, we present the first measurements of the magnetic spectral weight of optimally doped $\mathrm{Bi}_{2} \mathrm{Sr}_{2} \mathrm{CaCu}_{2} \mathrm{O}_{8+\delta}$ in absolute units. The lack of temperature dependence of the local spin susceptibility across the superconducting transition temperature, $T_{c}$ is incompatible with the itinerant calculations. Alternatively, the magnetic excitations could be due to local moments, as the magnetic spectrum is similar to that in $\mathrm{La}_{1.875} \mathrm{Ba}_{0.125} \mathrm{CuO}_{4}$ (ref. 15), where quasiparticles ${ }^{16}$ and local moments ${ }^{17}$ coexist.
\end{abstract}

$\mathrm{Bi}_{2} \mathrm{Sr}_{2} \mathrm{CaCu}_{2} \mathrm{O}_{8+\delta}$ has been the cuprate system of choice for surface-sensitive techniques such as angle-resolved photoemission spectroscopy (ARPES) and scanning tunnelling spectroscopy (STS) because it cleaves easily, thus enabling simple preparation of fresh surfaces. ARPES (refs 1-3) and STS (ref. 4) studies of $\mathrm{Bi}_{2} \mathrm{Sr}_{2} \mathrm{CaCu}_{2} \mathrm{O}_{8+\delta}$ have convincingly demonstrated the existence of coherent electronic excitations (Bogoliubov quasiparticles) in the superconducting state. The excitation gap for quasiparticles has $d$-wave symmetry, going to zero at four nodal points along the nominal Fermi surface in the two-dimensional reciprocal space for $\mathrm{C} \mathrm{CuO}_{2}$ plane ${ }^{1-3}$. On warming into the normal state, coherent electronic states are observed, at most, only over finite arcs about the nodal points; in the 'antinodal' regions, there is a so-called pseudogap and an absence of quasiparticles ${ }^{1,2}$.

The ARPES results on $\mathrm{Bi}_{2} \mathrm{Sr}_{2} \mathrm{CaCu}_{2} \mathrm{O}_{8+\delta}$ have been used as a basis for predicting the magnetic excitation spectrum in the superconducting state, measurable by inelastic neutron scattering ${ }^{10,14}$. To detect the magnetic signal with neutrons, however, one needs crystals of large volume to compensate for limited neutron source strength and weak scattering cross-section, and such crystals have been difficult to grow. The magnetic spectral weight is typically presented as the imaginary part of the dynamical spin susceptibility, $\chi^{\prime \prime}(\mathbf{Q}, \omega)$; here $\mathbf{Q}$ is the wave vector of a magnetic excitation and $E=\hbar \omega$ is its energy, with $\hbar$ being Planck's constant divided by $2 \pi$ and $\omega$ the angular frequency. Previous neutron scattering studies ${ }^{11-14}$ of $\mathrm{Bi}_{2} \mathrm{Sr}_{2} \mathrm{CaCu}_{2} \mathrm{O}_{8+\delta}$, working with crystals of limited size, focused on the change in $\chi^{\prime \prime}(\mathbf{Q}, \omega)$ on cooling through the superconducting transition temperature, $T_{\mathrm{c}}$.
A sustained effort has finally yielded crystals of sufficient size ${ }^{18}$ to enable a direct measurement of $\chi^{\prime \prime}(\mathbf{Q}, \omega)$ on an absolute scale. The as-grown crystals correspond to the condition of 'optimal' doping (maximum $T_{\mathrm{c}}$ ), with $T_{\mathrm{c}}=91 \mathrm{~K}$. To describe our results, we will follow previous practice ${ }^{11-14}$ and make use of a pseudo-tetragonal unit cell, with lattice parameters $a=3.82 \AA$ (parallel to in-plane $\mathrm{Cu}-\mathrm{O}$ bonds) and $c=30.8 \AA$. Wave vectors $\mathbf{Q}$ are given in reciprocal lattice units of $(2 \pi / a, 2 \pi / a, 2 \pi / c)$. The antiferromagnetic wave vector within a $\mathrm{CuO}_{2}$ plane corresponds to $\mathbf{Q}_{\mathrm{AF}}=(1 / 2,1 / 2)$. We note that the crystallographic unit cell is larger and orthorhombic, with a long-period incommensurate modulation in one in-plane direction. The substantial atomic displacements associated with the incommensurability can modulate electronic and magnetic interactions ${ }^{19}$; however, we did not detect any anisotropy of the magnetic scattering associated with the incommensurate modulation direction.

The magnetic response was measured by inelastic neutron scattering using a time-of-flight (TOF) technique (see the Methods section). Figure 1 shows constant-energy slices of the scattered signal as a function of momentum transfer about $\mathbf{Q}_{\mathrm{AF}}$ for several energies and temperatures. The results are plotted in the form of $\chi^{\prime \prime}(\mathbf{Q}, \omega)$, as discussed in the Methods section. Besides the magnetic scattering, peaked at $\mathbf{Q}_{\mathrm{AF}}$, the measurements also include contributions from phonons; the phonon contribution should be approximately independent of temperature, but should vary, on average, as $Q^{2}$. In this figure, we have subtracted a fitted background of the form $c_{0}+c_{2} Q^{2}$, where the coefficients $c_{0}$ and $c_{2}$ depend on energy, with $c_{2}$ independent of temperature. (The magnitude of the background can be seen in Fig. $2 \mathrm{a}-\mathrm{c}$, where plots of the measured intensity along specific directions in $\mathbf{Q}$ are plotted, with the fitted background indicated by a dashed line.) The magnetic signal is expected to be weaker and more diffuse at $310 \mathrm{~K}$, and the signal found there is generally consistent with this expectation. The diagonal streak of scattering for $\hbar \omega=36 \mathrm{meV}$ at $310 \mathrm{~K}$ indicates a phonon contribution with intensity modulated in $\mathbf{Q}$.

For a more quantitative analysis of the magnetic signal and its temperature dependence, we can take cuts through these images and fit Gaussian peaks to the structures. Examples of such plots are shown in Figs $2 \mathrm{a}-\mathrm{c}$ and 3 . From the fits, we obtain both the magnitude and $\mathbf{Q}$ dependence of the magnetic response. The effective dispersion of the magnetic excitations is plotted in Fig. 2e. Within the uncertainties, it is effectively isotropic about $\mathbf{Q}_{\mathrm{AF}}$. At low temperature, the overall shape of the dispersion is qualitatively consistent with the hour-glass spectrum that seems to be universal among the cuprates ${ }^{20}$, with the excitations crossing $\mathbf{Q}_{\mathrm{AF}}$ at $\hbar \omega \sim 40 \mathrm{meV}$ (ref. 14); the response falls off

\footnotetext{
${ }^{1}$ Brookhaven National Laboratory, Upton, New York 11973-5000, USA, ${ }^{2}$ Laboratoire Léon Brillouin, CEA-CNRS, CEA-Saclay, 91191 Gif sur Yvette, France, ${ }^{3}$ ISIS Facility, STFC Rutherford Appleton Laboratory, Chilton, Didcot, OX11 OQX, UK, ${ }^{4}$ Department of Physics, University College London, Gower Street, London WC1E 6BT, UK, ${ }^{5}$ CEA-Grenoble, INAC-SPSMS-MDN, 17 rue des Martyrs, 38054 Grenoble Cedex 9, France. *e-mail: jtran@bnl.gov.
} 


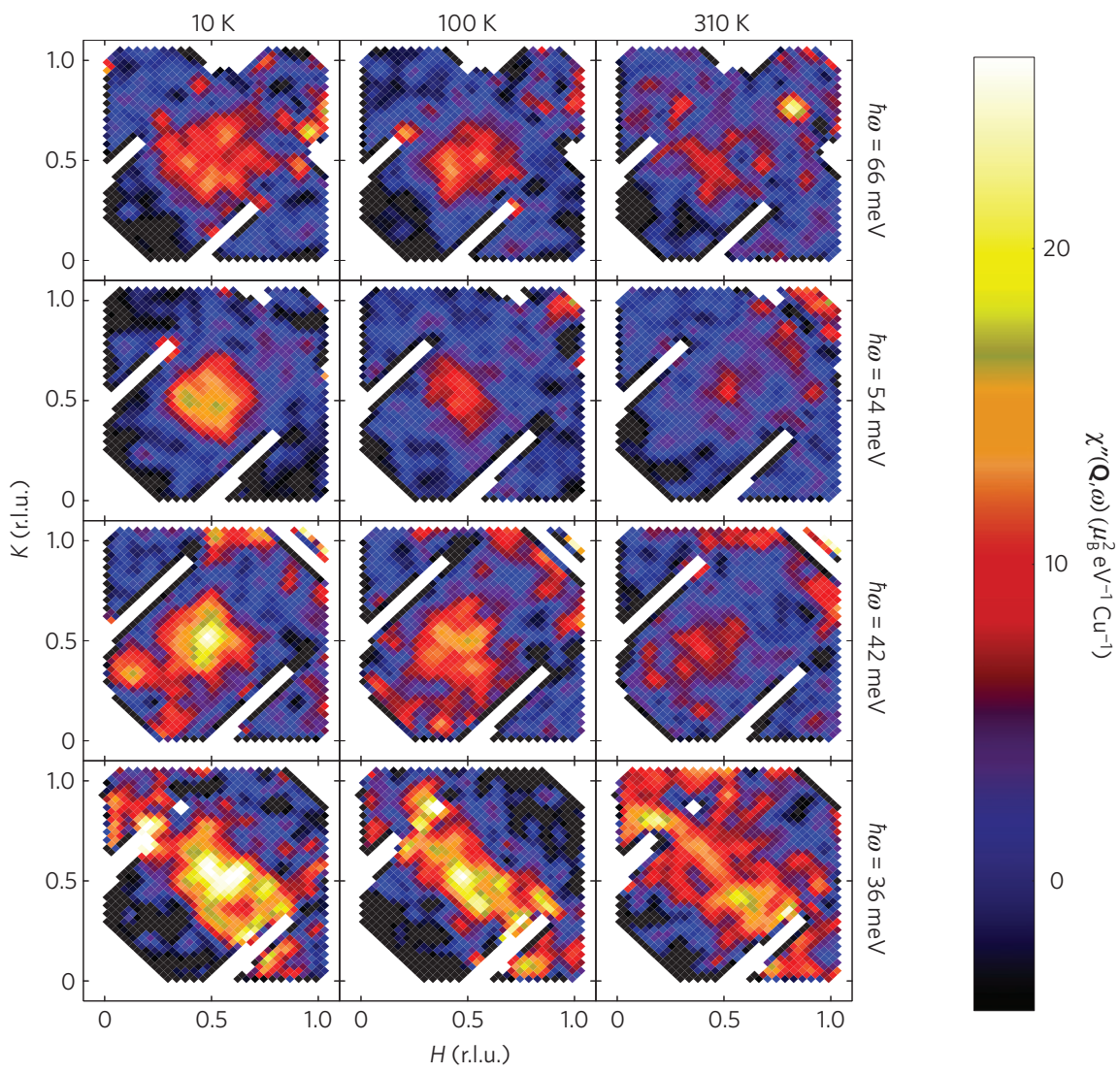

Figure 1 | Constant-energy slices through the scattered signal. TOF data (for $E_{\mathrm{i}}=120 \mathrm{meV}$ ) converted to the form of $\chi^{\prime \prime}(\mathbf{Q}, \omega)$ (see the Methods section), with background subtracted (see text). Data from four equivalent zones have been averaged to improve the statistics. Each column corresponds to a different temperature: 10, 100 and $310 \mathrm{~K}$, from left to right. Each row shows a different energy transfer: $36,42,54$ and $66 \mathrm{meV}$, from bottom to top. For each slice, the data have been averaged over an energy range of $\pm 3 \mathrm{meV}$. White indicates areas not covered by detectors.

below a spin gap energy of $\sim 25 \mathrm{meV}$ (see Fig. 3). The effective upward dispersion, above $40 \mathrm{meV}$, is comparable to that observed ${ }^{21}$ in underdoped $\mathrm{YBa}_{2} \mathrm{Cu}_{3} \mathrm{O}_{6.5}$, and much steeper than that ${ }^{22}$ in optimally doped $\mathrm{YBa}_{2} \mathrm{Cu}_{3} \mathrm{O}_{6.95}$.

The method that we have used to identify the magnetic response and separate it from the large phonon background is dependent on assumptions. To get a direct measure of the magnetic response, we have carried out a second experiment with spin-polarized neutrons (see the Methods section and Supplementary Information). As a result of intensity limitations associated with this technique, our useful results are limited to measurements at $\mathbf{Q}_{\mathrm{AF}}$; these are presented in Fig. 4a. The lower panels compare results for $\chi^{\prime \prime}\left(\mathbf{Q}_{\mathrm{AF}}, \omega\right)$ extracted from fits to the TOF data. The data in Fig. $4 a, c$ are generally consistent, with a substantial temperaturedependent change in signal at $\hbar \omega \sim 40 \mathrm{meV}$. More modest changes with temperature are seen in Fig. $4 \mathrm{~b}$, which corresponds to measurements with a different incident neutron energy, $E_{\mathrm{i}}$.

To understand the sensitivity to $E_{\mathrm{i}}$, we have to consider the fact that $\mathrm{CuO}_{2}$ planes in $\mathrm{Bi}_{2} \mathrm{Sr}_{2} \mathrm{CaCu}_{2} \mathrm{O}_{8+\delta}$ come in correlated bilayers, just as in $\mathrm{YBa}_{2} \mathrm{Cu}_{3} \mathrm{O}_{6+x}$. The magnetic response from a pair of layers can be separated into components with symmetry that is odd or even with respect to exchange of the layers. The relative cross-sections for the odd and even responses depend on the momentum transfer component along the $c$ axis, $Q_{c}$. For the TOF measurements, $Q_{c}$ varies with $\hbar \omega$ in a fashion that is determined by the choice of $E_{\mathrm{i}}$ (ref. 21). The relative weight of the odd contribution as a function of $E_{\mathrm{i}}$ and $\hbar \omega$ is indicated by the dashed grey lines in Fig. 4; the sum of the weights for the odd and even components at any given $\hbar \omega$ is equal to one. For $E_{\mathrm{i}}=200 \mathrm{meV}$ (Fig. $4 \mathrm{c}$ ), the response at $\hbar \omega \sim 40 \mathrm{meV}$ is mostly odd. For the polarized-neutron study, all of the measurements were done at a fixed wave vector that maximizes the odd component. For $E_{\mathrm{i}}=120 \mathrm{meV}$ (Fig. $4 \mathrm{~b}$ ), there is a bigger contribution from the even component, which shows less change with temperature ${ }^{13}$.

The enhancement of the odd component of $\chi^{\prime \prime}\left(\mathbf{Q}_{\mathrm{AF}}, \omega\right)$ in the superconducting state for $\hbar \omega \sim 40 \mathrm{meV}$ is consistent with previous work ${ }^{11,14}$; however, our absolute measurements of the magnetic response also indicate significant weight in the normal state. To get another perspective on the temperature dependence, we have integrated the fits to the magnetic response over the in-plane momentum transfers (assuming $\chi^{\prime \prime}(\mathbf{Q}, \omega)$ to be isotropic in $\left.\mathbf{q}=\mathbf{Q}-\mathbf{Q}_{\mathrm{AF}}\right)$ to yield the local susceptibility, $\chi^{\prime \prime}(\omega)$, plotted in Fig. $4 \mathrm{~d}-\mathrm{f}$. As can be seen, there are minimal changes in $\chi^{\prime \prime}(\omega)$ between 10 and $100 \mathrm{~K}$. Thus, the changes in $\chi^{\prime \prime}\left(\mathbf{Q}_{\mathrm{AF}}, \omega\right)$ seem to be associated with a narrowing of the $\mathbf{Q}$ dependence at low temperature.

Our results cast considerable doubt on the common view of the $\sim 40-\mathrm{meV}$ 'resonance' mode as a spin-1 excitation of a pair of quasiparticles in the superconducting state ${ }^{10}$, where the excitation is presumed to be resonant or excitonic because it occurs at an energy below twice the maximum superconducting $d$-wave gap energy (2 $\Delta$ ). From ARPES (ref. 3) and STS (ref. 4) studies, we know that $2 \Delta \approx 80 \mathrm{meV}$ for optimally doped $\mathrm{Bi}_{2} \mathrm{Sr}_{2} \mathrm{CaCu}_{2} \mathrm{O}_{8+\delta}$. It has been established that the superconducting gap decreases to zero at $T=T_{\mathrm{c}}$ for wave vectors on the nodal $\mathrm{arc}^{3}$. A pseudogap remains at antinodal wave vectors for $T>T_{c}$, but this involves only a weak depression of the electronic spectral function at the Fermi energy ${ }^{5}$. Thus, if the magnetic excitations were due to quasiparticles, we should have seen marked changes in $\chi^{\prime \prime}(\omega)$ between 10 and $100 \mathrm{~K}$ over the entire 

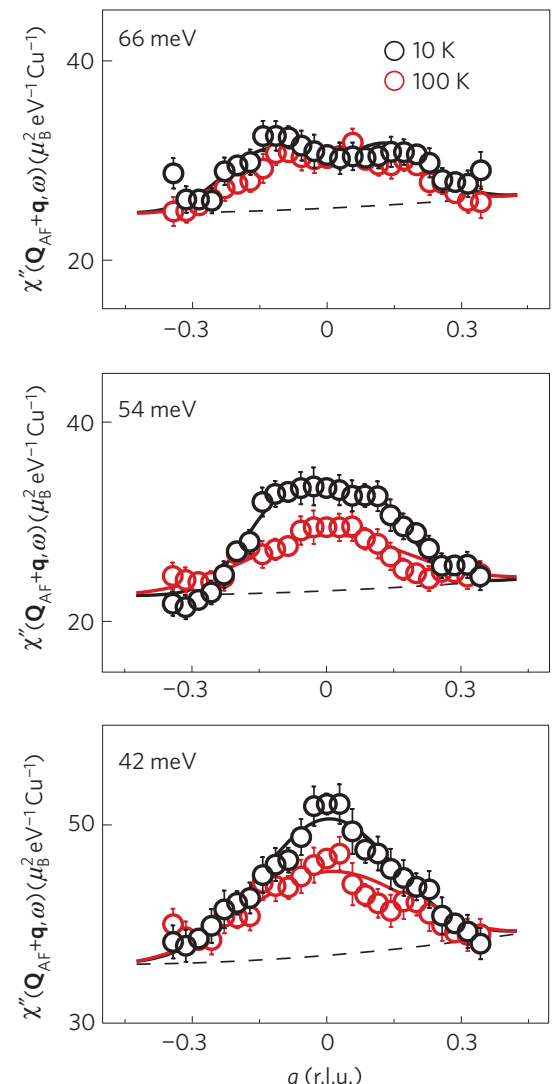

d
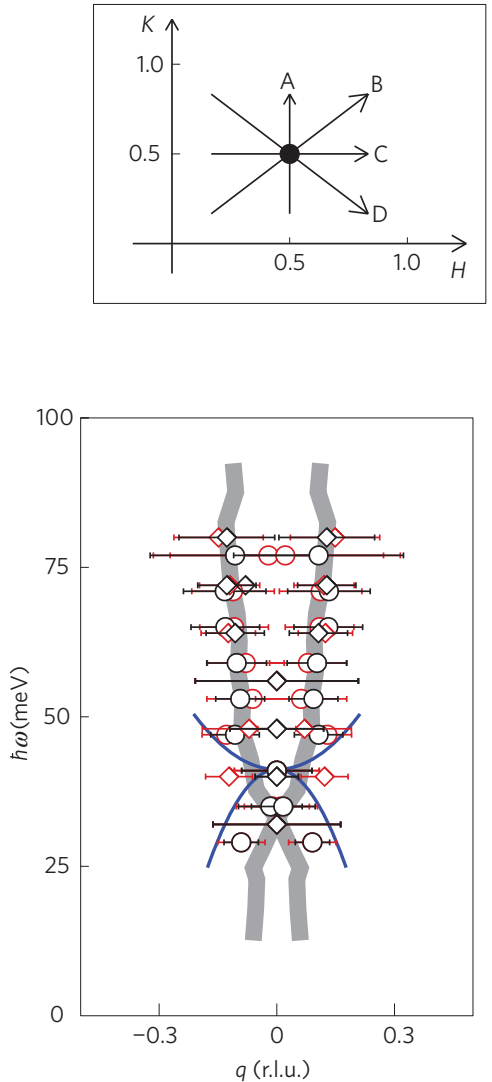

Figure 2 | Analysis of magnetic response. a-c, Cuts through $\chi^{\prime \prime}(\mathbf{q}, \omega)$ (for $E_{\mathrm{i}}=120 \mathrm{meV}$ ) along $\mathbf{q}=\mathbf{Q}-\mathbf{Q} \mathbf{A F}_{1}$ averaged over $\mathbf{q}$ along $[1,0,0]$ and $[0,1,0]$

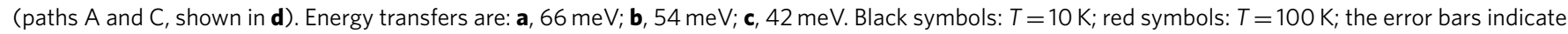
standard deviations. The solid lines through the data represent fitted Gaussian peaks-either a single peak or a pair of peaks constrained to be symmetric about $\mathbf{q}=0$. The dashed lines indicate background. $\mathbf{d}$, Schematic plot indicating directions of line cuts used for the least-squares peak-fitting analysis. e, Plot of peak positions obtained from the peak fitting. Circles: $E_{i}=120$ meV; diamonds: $E_{i}=200$ meV; the bars indicate peak widths. For $\hbar \omega>40$ meV, points represent fits to the averaged $A$ and $C$ cuts; virtually identical results were obtained for $B$ and $D$ cuts. For $\hbar \omega<40$ meV, only fits to $D$ cuts (as in Fig. 3) were used, because of the more complicated phonon background. The solid blue lines indicate magnetic dispersion for $\mathrm{YBa}_{2} \mathrm{Cu}_{3} \mathrm{O}_{6.95}$ from Reznik et al. ${ }^{22}$; the grey lines indicate results for $\mathrm{YBa}_{2} \mathrm{Cu}_{3} \mathrm{O}_{6.5}$ from Stock et al. ${ }^{21}$.

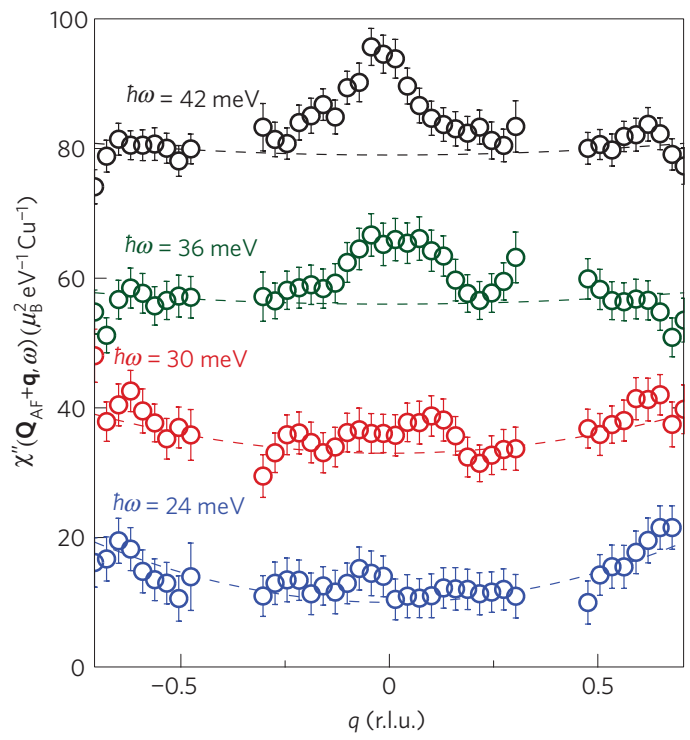

Figure 3 | Magnetic response at lower energies. Cuts through $\chi^{\prime \prime}(\mathbf{q}, \omega)$ along $\mathbf{q}=[1,-1,0]$ (path D in Fig. 2d) for excitation energies of 24, 30, 36 and $42 \mathrm{meV}$ (bottom to top) measured at $T=10 \mathrm{~K}$ with $E_{\mathrm{i}}=120 \mathrm{meV}$. Data sets have been offset vertically for clarity. The dashed lines indicate background. The error bars indicate standard deviations. measured energy range ${ }^{23}$; clearly, our results are inconsistent with such a picture. Similar inconsistencies for an electron-doped cuprate have been emphasized by Krüger et al. ${ }^{24}$. Furthermore, the strong temperature dependence of $\chi^{\prime \prime}(\omega)$ inferred from a conventional quasiparticle-based analysis of optical conductivity data $^{9}$ is inconsistent with our direct measurement, raising a challenge to that analysis.

The magnitude of $\chi^{\prime \prime}(\omega)$ in the energy range of $40-50 \mathrm{meV}$ (Fig. $4 \mathrm{~d}-\mathrm{f}$ ) is smaller than, but within a factor of two of, that for $\mathrm{YBa}_{2} \mathrm{Cu}_{3} \mathrm{O}_{6.95}$ (ref. 25), $\mathrm{La}_{1.84} \mathrm{Sr}_{0.16} \mathrm{CuO}_{4}$ (ref. 26) and $\mathrm{La}_{1.875} \mathrm{Ba}_{0.125} \mathrm{CuO}_{4}$ (ref. 15). In the case of $\mathrm{La}_{2-x} \mathrm{Ba}_{x} \mathrm{CuO}_{4}$, it has been shown that the magnetism is dominated by local moments on $\mathrm{Cu}$ atoms ${ }^{17}$. Given the comparable magnitude of the magnetic response, universal dispersion and the limited sensitivity to the presence of a superconducting gap, it seems likely that the spin fluctuations in $\mathrm{Bi}_{2} \mathrm{Sr}_{2} \mathrm{CaCu}_{2} \mathrm{O}_{8+\delta}$ are due primarily to localmoment magnetism. A recent study of $\mathrm{YBa}_{2} \mathrm{Cu}_{3} \mathrm{O}_{6.95}$ reached a similar conclusion ${ }^{23}$.

$\mathrm{La}_{1.875} \mathrm{Ba}_{0.125} \mathrm{CuO}_{4}$ may seem an unlikely case to compare with optimally doped $\mathrm{Bi}_{2} \mathrm{Sr}_{2} \mathrm{CaCu}_{2} \mathrm{O}_{8+\delta}$, as the former compound exhibits charge and spin stripe order ${ }^{17,27}$. Nevertheless, a recent ARPES study $^{16}$ has demonstrated the coexistence of nodal quasiparticles with the localized electronic moments of the spin stripes ${ }^{17}$, and there is evidence for two-dimensional superconducting correlations, as well ${ }^{28}$. Explaining such counterintuitive behaviour remains a challenge for theorists. 
a

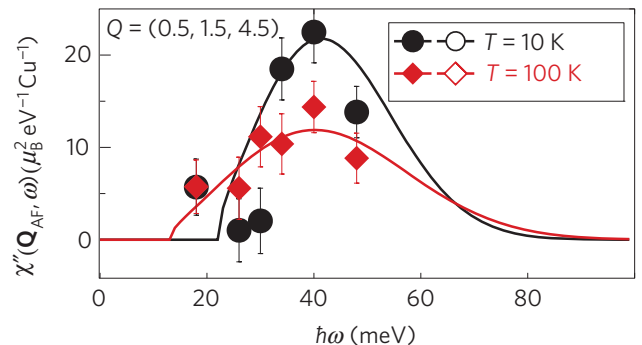

b

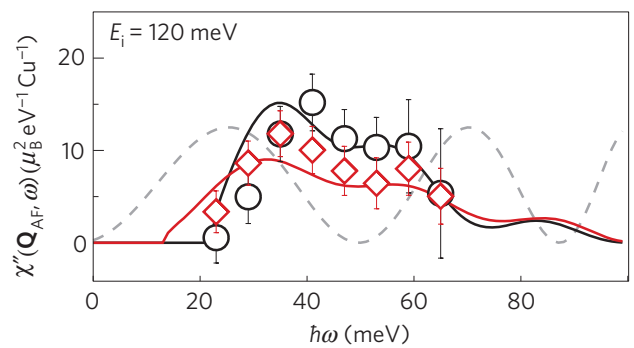

c

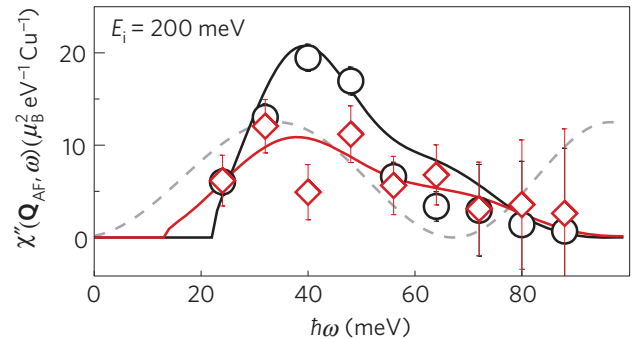

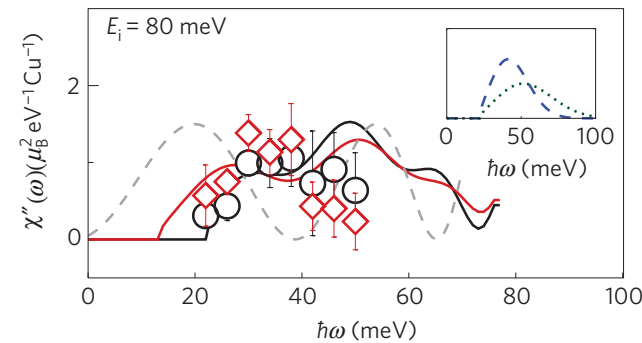
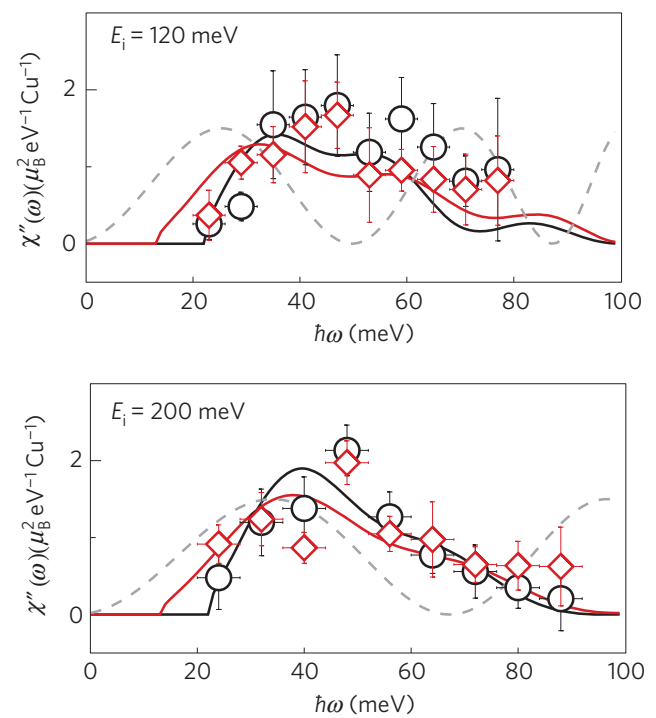

Figure 4 | Temperature and energy dependence of $\chi^{\prime \prime}$. a-c, $\chi^{\prime \prime}(\mathbf{Q}, \omega)$ at $\mathbf{Q}=\mathbf{Q}_{\mathrm{AF}}$, with data obtained from: spin-polarized beam measurements at $\mathbf{Q}=(0.5,1.5,4.5)(\mathbf{a}) ;$ TOF measurements with $E_{\mathrm{i}}=120 \mathrm{meV}(\mathbf{b})$; TOF data with $E_{\mathrm{i}}=200$ meV (c). The error bars indicate standard deviations. $\mathbf{d}-\mathbf{f}$, Local

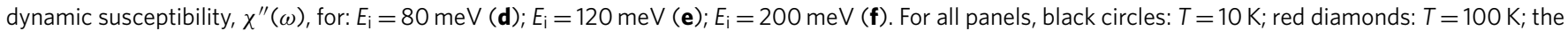
vertical bars indicate standard deviations, based on the least-squares fits used to evaluate and integrate the $\mathbf{Q}$ dependence of $\chi^{\prime \prime}(\omega, \mathbf{Q})$, whereas the horizontal bars indicate the energy range over which the signal was averaged. The inset in $\mathbf{d}$ shows a rough estimate of $\chi^{\prime \prime}(\omega)$ at $10 \mathrm{~K}$ for the odd (dashed) and even (dotted) components; at $100 \mathrm{~K}$, the gap is decreased, widths increased and areas kept approximately constant. These estimates are used (with

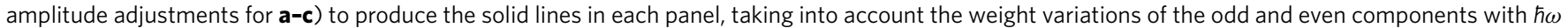
and $E_{\mathrm{i}}$; the grey dashed lines show the relative weight of the odd component (magnitude oscillates between 0 and 1 ).

\section{Methods}

The $\mathrm{Bi}_{2} \mathrm{Sr}_{2} \mathrm{CaCu}_{2} \mathrm{O}_{8+\delta}$ crystals were grown at Brookhaven using the travelling-solvent floating-zone method ${ }^{18}$. Plate-like single crystals with sizes up to $50 \times 7.2 \times 7 \mathrm{~mm}^{3}$ were obtained. Magnetic susceptibility measurements indicate an onset of diamagnetism at $T_{\mathrm{c}}=91 \mathrm{~K}$. Four crystals, with a total mass of $19 \mathrm{~g}$, were mounted on aluminium supports and co-aligned for the experiment on the MAPS TOF spectrometer at the ISIS spallation facility, Rutherford Appleton Laboratory. The sample was mounted in a closed-cycle He refrigerator for temperature control, with the $c$ axis oriented along the incident beam direction. The combinations of (incident neutron energy $E_{\mathrm{i}}$, Fermi chopper frequency, typical integrated beam current) used for the measurements were $(80 \mathrm{meV}, 250 \mathrm{~Hz}$, $5,000 \mu \mathrm{Ah}),(120 \mathrm{meV}, 350 \mathrm{~Hz}, 9,000 \mu \mathrm{A} \mathrm{h})$ and $(200 \mathrm{meV}, 500 \mathrm{~Hz}, 7,000 \mu \mathrm{A} \mathrm{h})$. Measured differential cross-sections, $\mathrm{d}^{2} \sigma / \mathrm{d} \Omega \mathrm{d} E$, were converted to absolute units by normalization to measurements on standard vanadium foils. The dynamic susceptibility was extracted using the formula

$$
\frac{\mathrm{d}^{2} \sigma}{\mathrm{d} \Omega \mathrm{d} E_{f}}=\frac{k_{f}}{k_{i}} \frac{1}{1-\mathrm{e}^{-\hbar \omega / k_{B} T}} f_{\mathrm{Cu}}^{2}(\mathbf{Q}) \chi^{\prime \prime}(\mathbf{Q}, \omega)
$$

where $f_{\mathrm{Cu}}(\mathbf{Q})$ is the anisotropic magnetic form factor for $\mathrm{Cu}$ (ref. 29).

A second set of seven crystals, with a mass of $18.75 \mathrm{~g}$, was aligned with X-rays at Brookhaven (collective mosaic $<2^{\circ}$ ) for the experiment on IN22 at the ILL. The spectrometer is equipped with a vertically focusing monochromator and horizontally focusing analyser, both made of Heusler alloy crystals. The sample, oriented with [100] and [039] directions in the horizontal scattering plane, was mounted in an ILL He-flow cryostat, inside Cryopadum, a system providing spherical polarimetry capabilities ${ }^{30}$. The neutron polarization flipping ratio measured on a strong Bragg peak was 16 . To obtain the inelastic magnetic scattering cross-section, we used an appropriate combination of spin-flip intensities measured for three orthogonal neutron polarizations (see Supplementary Information) with a fixed final energy of $30.5 \mathrm{meV}$. The results were normalized to the TOF data through non-spin-flip measurements of phonons.

\section{Received 9 February 2009; accepted 1 July 2009; published online 2 August 2009}

\section{References}

1. Yang, H. B. et al. Emergence of preformed Cooper pairs from the doped Mott insulating state in $\mathrm{Bi}_{2} \mathrm{Sr}_{2} \mathrm{CaCu}_{2} \mathrm{O}_{8+\delta}$. Nature 456, 77-80 (2008).

2. Kanigel, A. et al. Evidence for pairing above the transition temperature of cuprate superconductors from the electronic dispersion in the pseudogap phase. Phys. Rev. Lett. 101, 137002 (2008).

3. Lee, W. S. et al. Abrupt onset of a second energy gap at the superconducting transition of underdoped Bi2212. Nature 450, 81-84 (2007).

4. Kohsaka, Y. et al. How Cooper pairs vanish approaching the Mott insulator in $\mathrm{Bi}_{2} \mathrm{Sr}_{2} \mathrm{CaCu}_{2} \mathrm{O}_{8+\delta}$. Nature 454, 1072-1078 (2008).

5. Gomes, K. K. et al. Visualizing pair formation on the atomic scale in the high- $T_{\mathrm{c}}$ superconductor $\mathrm{Bi}_{2} \mathrm{Sr}_{2} \mathrm{CaCu}_{2} \mathrm{O}_{8+\delta}$. Nature 447, 569-572 (2007).

6. Lu, J. P. Neutron scattering as a probe for unconventional superconducting states. Phys. Rev. Lett. 68, 125-128 (1992).

7. Liu, D. Z., Zha, Y. \& Levin, K. Theory of neutron scattering in the normal and superconducting states of $\mathrm{YBa}_{2} \mathrm{Cu}_{3} \mathrm{O}_{6+x}$. Phys. Rev. Lett. 75, 4130-4133 (1995).

8. Bulut, N. \& Scalapino, D. J. Neutron scattering from a collective spin fluctuation mode in a $\mathrm{CuO}_{2}$ bilayer. Phys. Rev. B 53, 5149-5152 (1996).

9. Hwang, J., Timusk, T., Schachinger, E. \& Carbotte, J. P. Evolution of the bosonic spectral density of the high-temperature superconductor $\mathrm{Bi}_{2} \mathrm{Sr}_{2} \mathrm{CaCu}_{2} \mathrm{O}_{8+\delta}$. Phys. Rev. B 75, 144508 (2007).

10. Eschrig, M. The effect of collective spin-1 excitations on electronic spectra in high- $T_{\mathrm{c}}$ superconductors. Adv. Phys. 55, 47-183 (2006). 
11. Fong, H. F. et al. Neutron scattering from magnetic excitations in $\mathrm{Bi}_{2} \mathrm{Sr}_{2} \mathrm{CaCu}_{2} \mathrm{O}_{8+\delta}$. Nature 398, 588-591 (1999).

12. He, H. et al. Resonant spin excitation in an overdoped high temperature superconductor. Phys. Rev. Lett. 86, 1610-1613 (2001).

13. Capogna, L. et al. Odd and even magnetic resonant modes in highly overdoped $\mathrm{Bi}_{2} \mathrm{Sr}_{2} \mathrm{CaCu}_{2} \mathrm{O}_{8+\delta}$. Phys. Rev. B 75, 060502 (2007).

14. Fauqué, B. et al. Dispersion of the odd magnetic resonant mode in near-optimally doped $\mathrm{Bi}_{2} \mathrm{Sr}_{2} \mathrm{CaCu}_{2} \mathrm{O}_{8+\delta}$. Phys. Rev. B 76, 214512 (2007).

15. Tranquada, J. M. et al. Quantum magnetic excitations from stripes in copper oxide superconductors. Nature 429, 534-538 (2004).

16. He, R.-H. et al. Energy gaps in the failed high- $T_{\mathrm{c}}$ superconductor $\mathrm{La}_{1.875} \mathrm{Ba}_{0.125} \mathrm{CuO}_{4}$. Nature Phys. 5, 119-123 (2009).

17. Hücker, M., Gu, G. D. \& Tranquada, J. M. Spin susceptibility of underdoped cuprate superconductors: Insights from a stripe-ordered crystal. Phys. Rev. B 78, 214507 (2008).

18. Wen, J. S. et al. Large Bi-2212 single crystal growth by the floating-zone technique. J. Cryst. Growth 310, 1401-1404 (2008).

19. Slezak, J. A. et al. Imaging the impact on cuprate superconductivity of varying the interatomic distances within individual crystal unit cells. Proc. Natl Acad. Sci. USA 105, 3203-3208 (2008).

20. Birgeneau, R. J., Stock, C., Tranquada, J. M. \& Yamada, K. Magnetic neutron scattering in hole-doped cuprate superconductors. J. Phys. Soc. Jpn. 75, 111003 (2006).

21. Stock, C. et al. From incommensurate to dispersive spin-fluctuations: The high-energy inelastic spectrum in superconducting $\mathrm{YBa}_{2} \mathrm{Cu}_{3} \mathrm{O}_{6.5}$. Phys. Rev. $B$ 71, 024522 (2005)

22. Reznik, D. et al. Dispersion of magnetic excitations in optimally doped superconducting $\mathrm{YBa}_{2} \mathrm{Cu}_{3} \mathrm{O}_{6.95}$. Phys. Rev. Lett. 93, 207003 (2004).

23. Reznik, D. et al. Local-moment fluctuations in the optimally doped high- $T_{\mathrm{c}}$ superconductor $\mathrm{YBa}_{2} \mathrm{Cu}_{3} \mathrm{O}_{6.95}$. Phys. Rev. B 78, 132503 (2008).

24. Kruger, F. et al. Magnetic fluctuations in $n$-type high- $T_{\mathrm{c}}$ superconductors revea breakdown of fermiology: Experiments and Fermi-liquid/RPA calculations. Phys. Rev. B 76, 094506 (2007).
25. Woo, H. et al. Magnetic energy change available to superconducting condensation in optimally doped $\mathrm{YBa}_{2} \mathrm{Cu}_{3} \mathrm{O}_{6.95}$. Nature Phys. 2, 600-604 (2006).

26. Vignolle, B. et al. Two energy scales in the spin excitations of the high-temperature superconductor $\mathrm{La}_{2-x} \mathrm{Sr}_{x} \mathrm{CuO}_{4}$. Nature Phys. 3, 163-167 (2007).

27. Fujita, M., Goka, H., Yamada, K., Tranquada, J. M. \& Regnault, L. P. Stripe order, depinning, and fluctuations in $\mathrm{La}_{1.875} \mathrm{Ba}_{0.125} \mathrm{CuO}_{4}$ and $\mathrm{La}_{1.875} \mathrm{Ba}_{0.075} \mathrm{Sr}_{0.050} \mathrm{CuO}_{4}$. Phys. Rev. B 70, 104517 (2004).

28. Li, Q., Hucker, M., Gu, G. D., Tsvelik, A. M. \& Tranquada, J. M Two-dimensional superconducting fluctuations in stripe-ordered $\mathrm{La}_{1.875} \mathrm{Ba}_{0.125} \mathrm{CuO}_{4}$. Phys. Rev. Lett. 99, 067001 (2007).

29. Shamoto, S., Sato, M., Tranquada, J. M., Sternlieb, B. J. \& Shirane, G. Neutron-scattering study of antiferromagnetism in $\mathrm{YBa}_{2} \mathrm{Cu}_{3} \mathrm{O}_{6.15}$. Phys. Rev. $B$ 48, 13817-13825 (1993).

30. Regnault, L. P. et al. Spherical neutron polarization analysis on the three-axis spectrometer IN22. Physica B 350, E811-E814 (2004).

\section{Acknowledgements}

We gratefully acknowledge assistance from C. Stock, J. S. Wen and Z. J. Xu, and a critical reading of the manuscript by S. A. Kivelson. This work was supported by the Office of Science, US Department of Energy under Contract No. DE-AC02-98CH10886.

\section{Author contributions}

Sample preparation: G.D.G., M.H.; experiments: G.Y.X., B.F., T.G.P., L.P.R., J.M.T.; data analysis: G.Y.X., B.F.; paper writing: J.M.T., G.Y.X.

\section{Additional information}

Supplementary information accompanies this paper on www.nature.com/naturephysics. Reprints and permissions information is available online at http://npg.nature.com/ reprintsandpermissions. Correspondence and requests for materials should be addressed to J.M.T. 